\title{
Micromechanics-Based Stress Rupture Life Prediction Of Polymer Composites
}

\author{
T.J. Bandorawalla' and S.W. Case ${ }^{2}$ \\ Department of Engineering Science and Mechanics \\ Virginia Polytechnic Institute and State University \\ Blacksburg, VA 24061 USA
}

\begin{abstract}
This paper develops a Monte Carlo simulation technique to predict the stress-rupture lifetime of longitudinally loaded unidirectional polymer composites based on fiber and matrix properties. Matrix viscoelasticity is cited as the primary cause of rupture failure. Time-dependent matrix deformation leads to an increase in the overstressed length of unbroken fibers in the vicinity of a cluster of fiber fractures. A general time-dependent load-sharing framework that is able to account for an arbitrary sequence of fiber fractures is developed. Matrix deformations are based on the shearlag assumption. The time-dependent load sharing is incorporated into a Monte Carlo simulation for stressrupture lifetime. Even though the only material variability included in the simulation is the fiber strength distribution, very broad lifetime distributions are computed. The reasons for broad rupture lifetime distributions are discussed. It is shown that the strengthlife equal rank assumption does not apply for unidirectional polymer composites loaded in longitudinal tension because of fundamental differences between quasi-static and stress-rupture failure behavior. Encouraging comparisons are made to the experimental rupture lifetime of carbon fiber/polymer matrix composites.
\end{abstract}

Keywords: Creep, Static fatigue, Reliability, Probability, Fiber stress redistribution

\section{INTRODUCTION}

The use of composite materials in engineering applications requires an understanding of their behavior under various loading conditions. In particular, as composite materials are deployed in applications where several years of reliable service life are required, predictions of long-term durability are necessary. Because of the myriad of possible combinations of fiber and matrix materials, the best-case situation would be to make such predictions in terms of constituent properties. This is the goal of the present study for the case in which failure is governed by tensile failure of the fibers in the composite. A key aspect of this work is use of Monte Carlo simulations for lifetime prediction. There is ample experimental evidence that the rupture lifetime of a material is not deterministic. Hence, it is extremely important for life-prediction techniques to be able to determine component reliability at a given stress level for a desired lifetime. The models presented in this work utilize probabilistic techniques to account for variability in fiber strength that translate into variability in lifetime.

An important aspect of this work is time-dependent fiber stress redistribution, or load-sharing, in the vicinity of broken fibers. Hedgepeth /I/ and Hedgepeth and Van Dyke $12 /$ introduced influence-function techniques to model quasi-static load sharing. In this work timedependent models for load sharing are required by assuming linearly elastic properties for the fibers and linearly viscoelastic matrix properties. Lagoudas et. al.

\footnotetext{
'Presently with Intel Corporation

${ }^{2}$ Corresponding author: Virginia Polytechnic Institute and State University, 120 Patton Hall, Blacksburg, VA 24061. U.S.A. Ph: (540) 231 3140, Fax: (540) 231 9187, scase@vt.edu
} 
/3/ and Beyerlein et. al. /4/ have developed timedependent load-sharing models within the shear-lag framework. Lagoudas et. al. developed exact, closedform, and approximate load-sharing solutions for a single unidirectional composite lamina with a cluster of adjacent coplanar fiber fractures. They also extended their results to consider a growing cluster of coplanar breaks. Beyerlein et. al. developed a more general approach called viscous break interaction (VBI). They presented approximate and numerical techniques for arbitrarily positioned fiber fractures occurring simultaneously in a three-dimensional volume of material. Although Beyerlein et. al. did suggest alterations to consider breaks that occurred sequentially in time, they did not present a complete analysis. Moreover, both Lagoudas et. al. and Beyerlein et. al. assumed a power law creep compliance model for the matrix material. In this work we present a general approximate numerical framework for time-dependent load sharing that is able to account for arbitrarily positioned fiber fractures that occur sequentially in time. A direct Laplace inversion approximation introduced by Schapery $15 /$ is used for the analysis. This approximation makes it possible to accommodate a more general creep law that can be expressed as a Prony series. Section 2 discusses two different shear-lag based load-sharing methods: the traditional approach of Hedgepeth and Van Dyke, and a simplification where the load of a broken fiber is redistributed only onto its nearest neighbors. The two methods are compared to each other in Section 3. A qualitative comparison is also made to time-dependent strain concentration measurements on model composites $/ 6 /$.

Lifshitz and Rotem /7/ were the first to develop a micromechanical theory for stress-rupture of composite materials consisting of brittle fibers with probabilistic strengths embedded in a viscoelastic matrix. It was widely believed that stress-rupture of polymer composites resulted from the time-dependent strength of some fibers (e.g. glass fibers). Lifshitz and Rotem used their model to show that delayed failure can occur even when the fiber strengths are not timedependent. Since most of the literature dealt with stressrupture of glass-reinforced materials, Lifshitz /8/ conducted some preliminary tests to show that the stress-rupture phenomenon occurred in carbon- reinforced epoxies even though carbon fibers are widely believed to be free of any creep response. Section 3 presents further experimental and theoretical confirmation of the mechanism that leads to delayed failure of longitudinally loaded unidirectional polymer composites even when fiber strengths are not timedependent.

In this work we develop Monte Carlo simulations to model the stress-rupture process in composites with a viscoelastic matrix. Ibnabdeljalil and Phoenix /9/ have used similar Monte Carlo simulations to investigate the statistics of composite failure in brittle matrix composites resulting from fiber strength degradation. Along similar lines, Iyengar and Curtin $/ 10,11 /$ implemented Monte Carlo simulations for stress-rupture of metal and ceramic matrix composites due to the combined effect of strength degradation of the fibers, and matrix and interfacial shear creep. Iyengar and Curtin / $/ \mathrm{l} /$ used the formulation of $\mathrm{Du}$ and McMeeking $/ 12 /$ to model the effect of matrix creep resulting from time-dependent relaxation of normal and shear stresses in metal and ceramic matrix composites. Theoretical models have also been developed to predict stressrupture lifetimes of simple model composite systems with few fibers (Phoenix et. al., /13/; Otani et. al., /14/. Making comparisons of the model predictions with rupture lifetimes of carefully controlled model systems provides valuable insight into the rupture failure process that helps with modeling "real" composite systems.

Section 4 develops the general stress-rupture simulation framework for longitudinally loaded unidirectional polymer composites consisting of linearly elastic fibers with Weibull strengths and a linearly elastic matrix. Unidirectional polymer composites consistently fail in or near the gripped section of the specimen. In order to alleviate this problem stressrupture lifetimes are measured on an AS-4 carbon fiber/ polyetheretherketone (PEEK) laminate with $\left[90 / 0_{3}\right]_{s}$ layup. The $90^{\circ}$ surface plies carry a negligible fraction of the total load on the material and protect the inner core of load-carrrying $0^{\circ}$ plies. Quasi-static strengths and rupture lifetime measurements on the AS-4/PEEK composite system are presented. Comparisons between the rupture predictions and measurements on the AS4/PEEK system are also presented in Section 4. Finally 
in Section 5, the major results and conclusions are summarized.

\section{TIME-DEPENDENT LOAD SHARING}

Bandorawalla /6/ examined a quasi-static framework to calculate the micromechanical fiber stress redistribution due to arbitrary fiber facture locations. The technique was based in superposition of the effect of individual fiber fractures. The time-dependent version is considerably more complicated. The times at which fiber fractures occurred and their locations need to be taken into account to calculate the fiber stresses at any position and time. This general time-dependent framework for load-sharing is discussed in detail in the work of Bandorawalla. In summary, the fibers are assumed to be linearly elastic, while the matrix is assumed to be linearly viscoelastic. It is shown that the effect of each fiber fracture is expressed as a convolution of the crack tip opening-displacement at the fiber fracture location and a time-dependent force influence-function. The force influence-functions depend on geometric and material properties of the composite, and assumptions of the mechanism of load transfer (i.e. shear-lag, finite element) and number of neighboring fibers involved in this transfer. Once again we draw on experience with modeling quasi-static load sharing to compute the force influence-functions. Two different types of load sharing may be utilized, depending upon the technique used to calculate the force influence-functions. They are called Nearest Neighbor Load Sharing (NNLS) and Hedgepeth and Van Dyke Load Sharing (HVDLS). NNLS assumes that the load of a broken fiber is redistributed only onto the nearest neighbors. The authors believe that NNLS may be valid for time-dependent stress redistribution too. HVDLS is a time-dependent extension of the traditional quasi-static technique introduced by Hedgepeth and Van Dyke $12 \%$. In HVDLS the load of a broken fiber is transferred onto all the surrounding fibers with preferential load transfer to the nearer fibers. A comparison between the time-dependent stresses calculated by the two approaches is made in Section 3 .

\section{COMPARISON BETWEEN NNLS AND HVDLS}

Representative stress profiles in neighboring fibers caused by an isolated break in fiber $(0,0)$ at $x-0$ are shown in Figure 1 through Figure 4. The axial location along the fiber is expressed in terms of the fiber radius, $r_{f}$. Under NNLS only the stress in the nearest neighbor is perturbed as shown in Figure 1. Although the far-field fiber stress is held constant, matrix viscoelasticity causes the overloaded length on unbroken fibers adjacent to a fiber fracture location to increase with time. Consequently, there is a greater probability of fiber failure occurring in these unbroken fibers. This time-dependent fiber stress redistribution is the primary cause of failure in a unidirectional polymer matrix composite under longitudinal stress-rupture loading. Similar trends in the time dependence of stresses calculated by HVDLS are seen in Figure 2 through Figure 4. The HVDLS results are computed for a $10 \times 10$ array of hexagonally packed fibers. HVDLS predicts a lower peak stress concentration than NNLS in the fibers closest to the fractured fiber. However, the HVDLS approach also produces a small perturbation of the stresses in the next to nearest neighbors as shown in Figure 3 and Figure 4 . The peak stress concentration on the next to nearest neighboring fibers is much smaller than on the nearest neighbors. An important consequence of shear-lag assumptions is that the peak stress concentration due to an isolated fiber fracture does not change with time. In fact this peak stress concentration is not a function of any geometric or material properties of the composite if regular hexagonal fiber packing is assumed. It should also be pointed out that since under shear-lag assumptions the matrix does not carry any normal tensile stress there is no provision in the analysis to account for an increase in the far-field fiber stress with time due a viscoelasticitybased decrease of the tensile load carried by the matrix. This is not a serious source of error since for most polymer matrix composites with high fiber volume fractions and high fiber to matrix stiffness ratios the total tensile load carried by the matrix is negligible.

The stresses in the broken fiber decrease with time as shown in Figure 5. Although Figure 5 shows stresses calculated by NNLS, very similar curves are obtained 


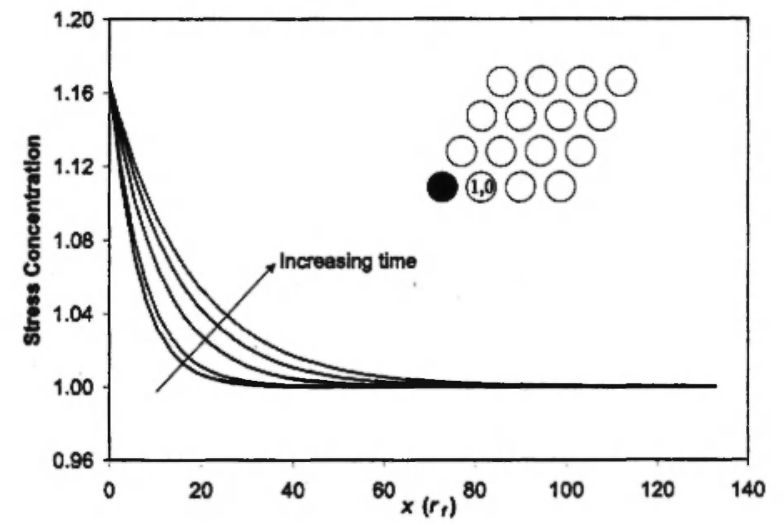

Fig. 1: Stress in fiber $(1,0)$ due to isolated break in shaded liber at $x=0$ computed with NNLS

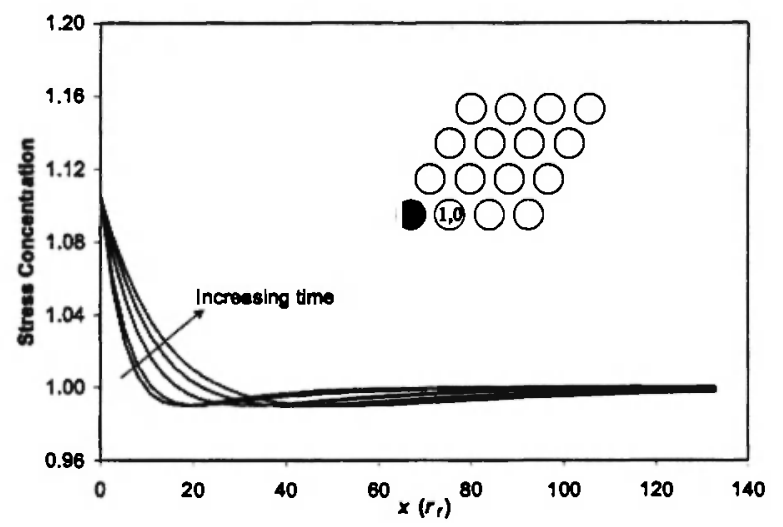

Fig. 2: Stress in fiber $(1,0)$ due to isolated break in shaded fiber at $x=0$ computed with HVDLS

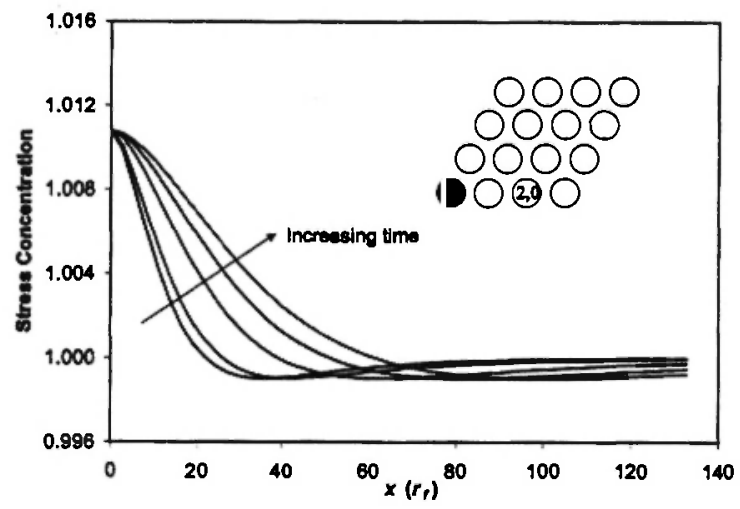

Fig. 3: Stress in fiber $(2,0)$ due to isolated break in shaded fiber at $x=0$ computed with HVDLS

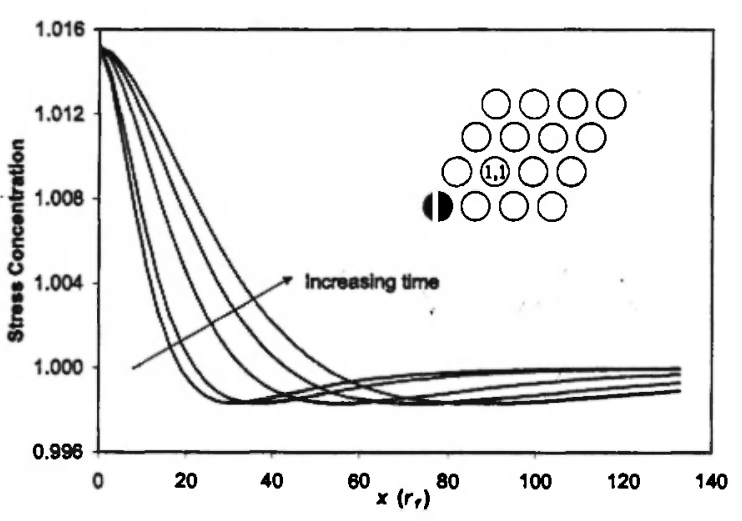

Fig. 4: Stress in fiber $(1,1)$ due to isolated break in shaded fiber at $x=0$ computed with HVDLS

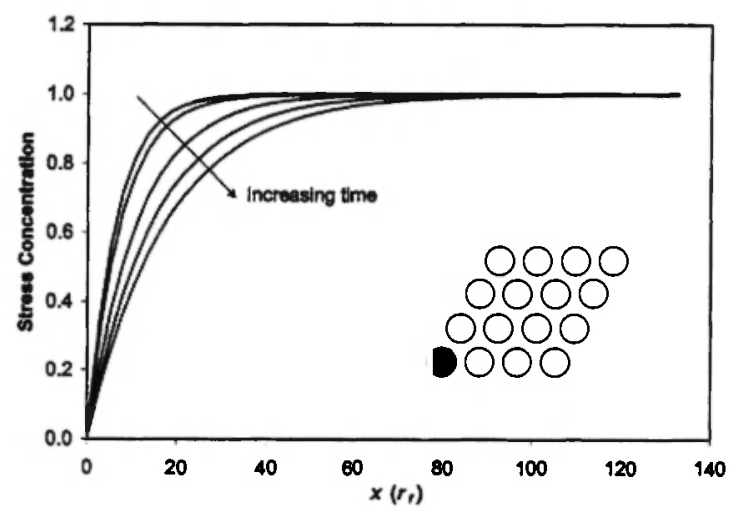

Fig. 5: Stress in broken fiber $(0,0)$ due to isolated break at $x=0$ computed with NNLS

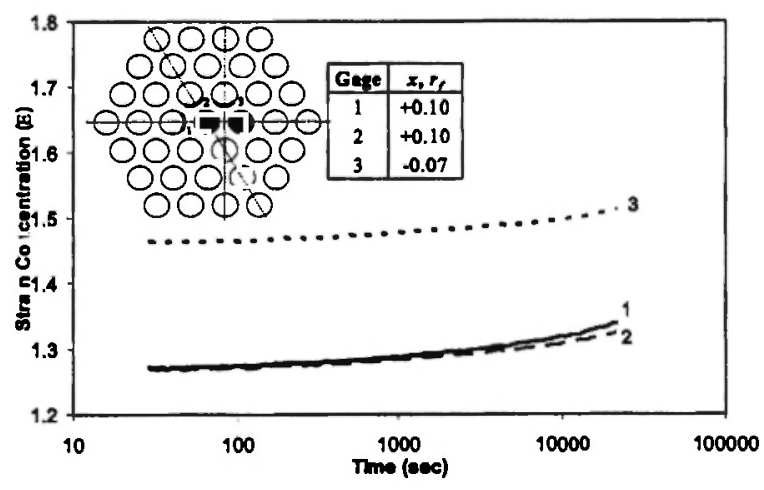

Fig. 6: Model composite measurements of strain concentrations due to a two adjacent coplanar fiber fractures 
for HVDLS. It is unlikely for another break to occur in the under-stressed region of a broken fiber. Hence, the decrease of axial stress in a broken fiber with time is not the controlling mechanism for stress-rupture failure in unidirectional polymer composites.

Bandorawalla $/ 6 /$ made in-situ fiber strain concentration measurements in macromodel composites with fibers that were large enough that strain gages could be mounted directly onto the surface of the fibers. The time-dependent strain concentration measurements agreed with the trends described in this paper for both the broken fibers and the unbroken neighbors. Representative measurements on unbroken neighboring fibers with two adjacent coplanar fiber fractures are shown in Figure 6. The axial positions of the gages referenced to the plane of fiber fractures are denoted by $x$ in Figure 6. The presence of multiple adjacent fiber fractures produces a more pronounced change in strain concentration with time than for an isolated fiber fracture. The model composite measurements provide a qualitative verification for the load sharing philosophy described in Section 2. A detailed time-dependent finite element analysis of the model composite domain is necessary to make a quantitative comparison between the measurements and modeling approach. The finite elcment model could then be used to establish whether NNLS or HVDLS is more appropriate for modeling time-dependent micromechanical stress redistribution in unidirectional composite materials. This procedure was followed by Bandorawalla $/ 6 /$ to investigate the applicability of shear-lag models for quasi-static load sharing.

\section{STRESS-RUPTURE LIFETIME MODELING}

A Monte Carlo simulation is used to predict the stress-rupture lifetime of a unidirectional composite material. Micromechanical stress redistribution can be calculated by applying either the NNLS or HVDLS desrribed in Section 2. Initial rupture lifetimes are measured on a unidirectional carbon fiber/polymer matrix composite. The difficulties associated with measuring rupture lifetimes of unidirectional systems are discussed, and rupture lifetimes are obtained for an alternate material system with $90^{\circ}$ plies on the surface. Comparisons between the simulation predictions and lifetime measurements on the alternate material system are presented.

\subsection{Rupture Simulation Approach}

Failure of unidirectional composite materials loaded in tension in the fiber direction is controlled by failure of fibers. The stochastic simulation approach attempts to track the progression of fiber fractures leading to eventual composite failure. All material property inputs to the simulation other than the fiber strength are assumed to be deterministic. A Weibull distribution given by

$$
P_{f}(\sigma, l)=1-\exp \left[-\frac{l}{l_{o}}\left(\frac{\sigma}{\sigma_{o}}\right)^{m}\right]
$$

describes the probability of failure $P_{f}$, of a fiber of length $l$, at a stress level $\sigma . \sigma_{0}$ is the Weibull location parameter, and $m$ is the fiber Weibull modulus or shape parameter. $\sigma_{0}$ is interpreted as the stress level required to cause one failure on average in a fiber of length $l_{v} . m$ is related to the variability in fiber strength, with a higher $m$ for a narrower distribution. Weibull parameters for the strength distribution of certain fibers are available in the literature.

An outline of the stress-rupture simulation procedure and the representative volume of material with fibers and matrix is shown in Figure 7. Uniform hexagonal fiber packing is assumed. In order to track the location

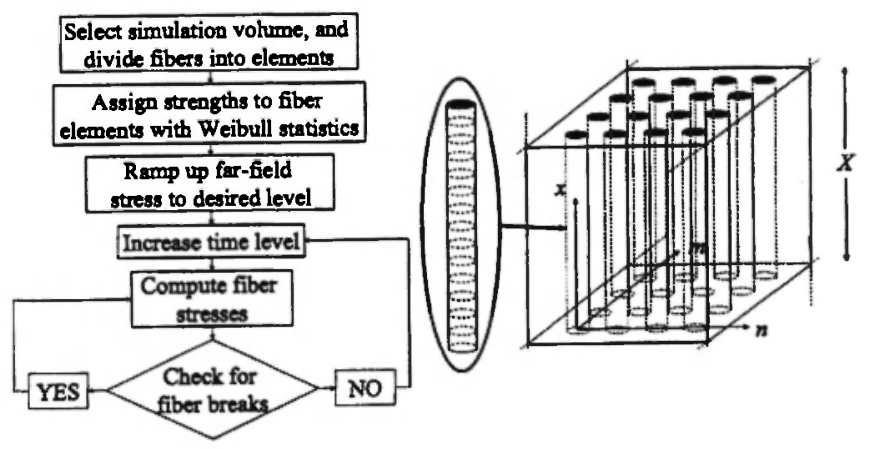

Fig. 7: Flowchart of Monte Carlo simulation for stress rupture lifetime with representative volume element (RVE) 
of fiber fractures every fiber is subdivided into the same number of elements along its length as shown in Figure 7. A fracture is allowed to occur at a random location within each fiber element. Landis et al. /15/ have reported that positioning a break at random within a fiber element significantly reduces the number of elements along each fiber required for convergence of the simulation results. Values of strength are assigned to the fiber elements by using the Weibull strength distribution of Equation (1). While the distribution of fiber strength remains the same, the actual element strengths change for every computation of rupture lifetime. To begin the simulation process, the far-field axial fiber stress $\sigma_{g}$, is increased to the fiber stress level at which rupture lifetimes are desired i.e. $\sigma_{i j}^{\text {rupt }}$. This initial ramp up is assumed to occur instantaneously, and depending on the rupture stress level may result in fiber element failures. During the initial ramp up, fiber stress redistribution is calculated by applying the quasi-static version of load sharing using the instantaneous matrix modulus since ramp up is assumed to be instantaneous and hence matrix viscoelasticity does not play a role. The general time-dependent load sharing framework is easily specialized to determine the instantaneous stress redistribution. This is achieved to compute fiber stresses at $t=0$ due to a single set of $b_{1}$ breaks that occur simultaneously at $t_{1}=0$. The ramp up is carried out by increasing the far-field stress to cause failure of the next weakest element only if no further fiber element failures occur due to stress redistribution at the current far-field stress level. Once $\sigma_{\tilde{v}}^{i u p i}$ is attained, the far-field fiber stress is held constant and the time level is incremented to cause failure of fiber elements. The time level is incremented in a geometric progression to maximum of $t=t_{\max }$. This results in a linear increase in time on a logarithmic scale. At each new time level fiber stresses are computed and a check is performed for further fiber element failures. If a fiber failure is detected at a current time level, it is assumed to have occurred at an intermediate time halfway between the previous time level and the current time level. Following the notation used by Bandorawalla $/ 6 /$, the most recent fiber failures occur at $t_{r}$. Local stress redistribution may result in further fiber element failures at the same time level. These additional failures are assumed to occur at $t_{n}$ and hence, $b_{r}$ may increase due to stress redistribution alone.
The time level is incremented only after no further fiber element failures occur at the same time level. The process is repeated until the surviving fiber elements in a cross-section of the simulation volume can no longer sustain the global load i.e. stress rupture material failure is predicted. The time level at this point is the calculated rupture lifetime of the simulation volume. It is also possible that stress rupture failure does not occur by $t_{\max }$, in which case a runout is predicted. In order to expedite the simulation process two techniques are implemented:

1. If all the fiber elements are intact after the initial ramp up, then the time-dependent load-sharing framework described in Section 2 will not predict any change in fiber stresses with time, and hence, no fiber element failures at all. This is a consequence of assuming that the fibers are linearly elastic and that the matrix is capable of sustaining only shear stresses. Hence, it is not necessary to progressively increase the time level as a runout will be predicted if no fiber element failures occur during the initial ramp up.

2. Even if a few fiber failures do occur during the initial ramp up, no additional fiber fractures may occur in $t_{\max }$ due to time-dependent fiber stress evolution. This is very easily checked by directly computing the stresses at $t_{\max }$ due to only those fiber fractures that occur during the initial ramp up. If the stresses at $t_{\max }$ are not large enough to cause failure of any additional fiber elements then a runout is predicted without having to progressively increase the time level.

3. Interpolation in both space and time is used for the force-influence functions. (For details, the reader is referred to Bandorawalla $/ 6 /$.)

Before the simulation procedure described above can be used to obtain lifetimes the far-field fiber stress level $\sigma_{\tilde{j}}^{i u j i}$ needs to be established. The first step in this process is to compute the composite strength distribution of the simulation volume. This is achieved by ramping up the far-field fiber stress instantaneously and calculating fiber stress redistribution with the quasistatic version of load sharing as described earlier in this section. The composite strength of the simulation volume corresponds to the far-field fiber stress at which 
all the fiber elements in a cross-sectional plane fail. One hundred strengths are computed in this manner. The computed composite strengths of the simulation volume conform to a Weibull distribution with a location and shape parameter given by $\tilde{\sigma}_{J}^{\text {sim }}$ and $\tilde{m}^{\text {sim }}$, respectively. The second step in establishing $\sigma_{f f}^{\text {rupt }}$ is to measure strengths of the composite material under consideration. The experimental composite strengths conforms to a Weibull distribution with location and shape parameter $\tilde{\sigma}_{o}^{\exp }$ and $\tilde{m}^{\exp }$, respectively. The experimental rupture lifetimes are measured at a composite stress level of $R^{\mathrm{cxp}} \tilde{\sigma}_{o}^{\exp }$. The composite stress level for performing the rupture simulations is $R^{\mathrm{vim}} \tilde{\sigma}_{\bar{i}}^{\mathrm{sim}} . R^{\mathrm{vim}}$ is calculated by equating the experimental instantaneous probability of failure of the composite at $R^{\text {cxp }} \tilde{\sigma}_{* ;}^{\text {aur }}$ to the instantaneous probability of failure of the simulation volume at $R^{\mathrm{sim}} \tilde{\sigma}_{t}^{\text {sim }}$. Thus,

$P_{f}=1-\exp \left[-\left(R^{\exp }\right)^{\check{r}^{\exp }}\right]=1-\exp \left[-\left(R^{\mathrm{sim}}\right)^{\tilde{m}^{\operatorname{sim}}}\right]$

Finally, the far-field fiber stress level for the rupture simulation is given by

$$
\sigma_{f f}^{\text {rupt }}=\frac{R^{\text {sim }} \tilde{\sigma}_{o}^{\text {sim }}}{V_{f}}
$$

\subsection{Material Systems}

Initial measurements of rupture lifetime are made on a Grafil 34-700 standard modulus/polyphenylene sulfide (PPS) pultruded unidirectional composite tape. The experimental composite strengths of the Grafil carbon fiber/PPS composite conform to a Weibull distribution with $\tilde{\sigma}_{0}^{\exp }=1.57 \mathrm{GPa}$ and $\tilde{m}^{\exp }=29.4$ at a gage length $\tilde{l}_{o}=76 \mathrm{~mm}$. The composites have a fiber volume fraction $V_{f}$, of $40 \%$. The experimental rupture lifetimes of the Grafil carbon fiber/PPS composite are shown in Figure 8 . When the stress rupture simulation was used to predict lifetime, two significant inconsistencies between experimental lifetimes and the predictions were observed:

1. The simulation methodology over-predicted stress rupture lifetimes, and

2. The rupture lifetime predictions had much greater variability than the experimental lifetimes.

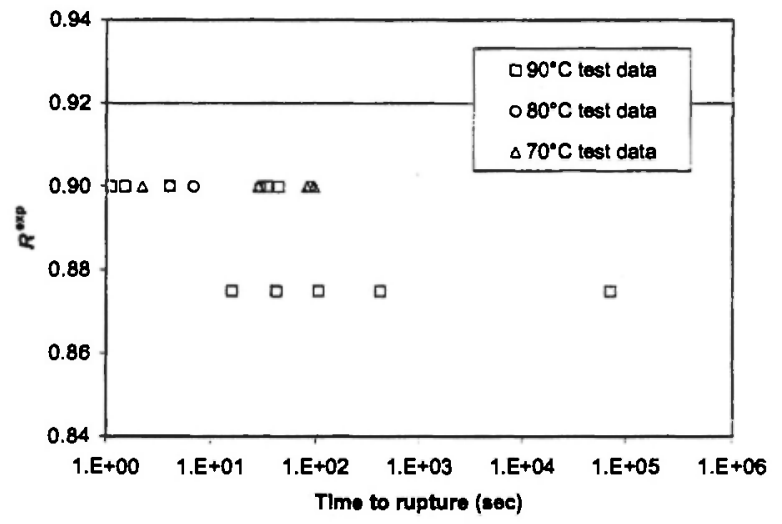

Fig. 8: Stress rupture lifetimes of Grafil carbon fiber/PPS unidirectional composite

Measuring the tensile strength and rupture lifetime of purely unidirectional composite materials poses certain challenges. Although the specimens are tabbed as shown in Figure 9, the application of grip pressure unavoidably causes material damage and fiber fractures in the gripped section of the specimen. The timedependent propagation of these defects in the grip section during stress rupture loading dominates the failure behavior of purely unidirectional specimens. The

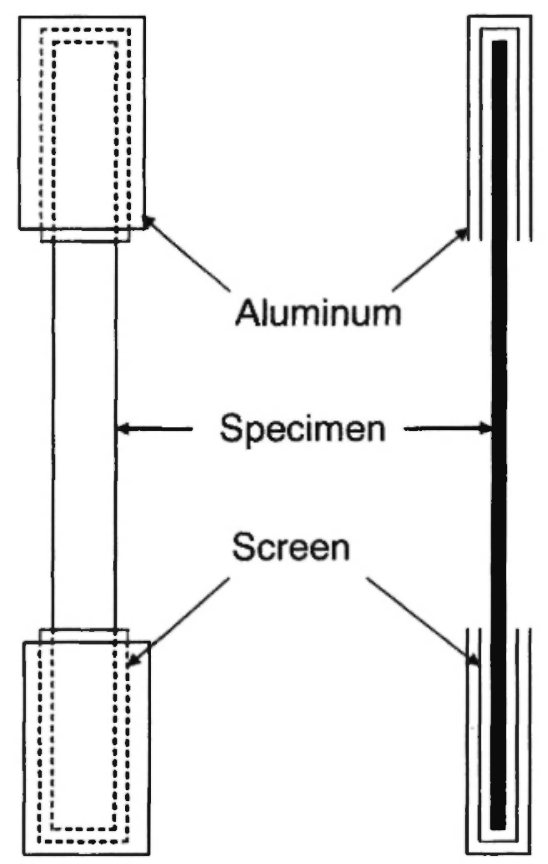

Fig. 9: Tabbing of specimens for tensile strength and stress rupture testing 
tabbing materials used consisted of 100-count (100 wires per linear inch) stainless steel screen and 1000 series aluminum sheet that was 0.02 inches thick. A piece of screen is folded in half over the ends of the specimen, and an aluminum piece is folder over the screen on the ends of the specimen as shown in Figure 9. It is difficult to adhesively bond tabbing material such as Grade G-10 Garolite woven glass fiber laminates to a PPS-based composite. Even with adhesively bonded tabs failures were observed in or near the gripped section due to a high stress concentration.

In order to alleviate the problems caused by gripinduced damage stress-rupture lifetimes are measured on an alternate material system with $90^{\circ}$ external plies. Composite panels with a $\left[90 / 0_{3}\right]_{\mathrm{s}}$ layup are compression molded from APC-2 prepreg supplied by Cytec Industries. APC-2 prepreg consists of AS4 carbon fiber with a thermoplastic polyetheretherketone (PEEK) matrix. The specimens have a fiber volume fraction of $54 \%$ and a gage length of $76 \mathrm{~mm}$ with a rectangular cross-section of $1 \mathrm{~mm} \times 12.7 \mathrm{~mm}$, nominally. The tabbing method described earlier in this section is used to test the APC-2 composite. However, a finer 200count (200 wires per linear inch) stainless steel screen was used instead of the 100-count screen. The finer tabbing screen and the $90^{\circ}$ external plies protect the load carrying $0^{\circ}$ plies from damage in the gripped section. Figure 10 shows a typical failed Grafil/PPS unidirectional specimen along with a typical failed APC-2 $/ 90 / 0_{3} / \mathrm{s}$ specimen. While it is difficult to pinpoint where initial and ultimate failure occurred in the Grafil/PPS unidirectional specimens, all the APC-2 $\left[90 / 0_{3}\right]_{\mathrm{s}}$ specimens consistently failed in the gage section.
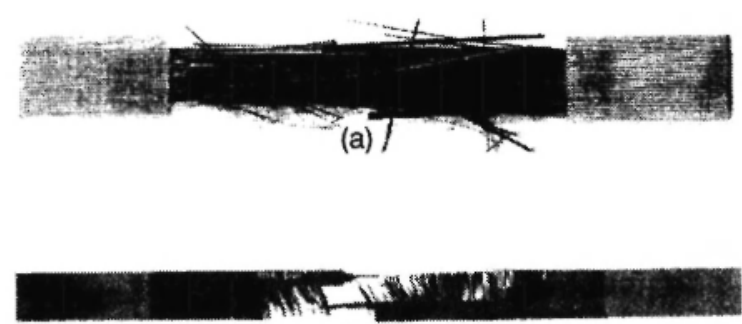

(b)

Fig. 10: Failed specimens. (a) Grafil carbon fiber/PPS unidirectional composite (b) APC-2 $/ 90 / 0_{3} / \mathrm{s}$ laminate
The relative stiffness of the $0^{\circ}$ and $90^{\circ}$ laminae in the direction of the tensile load is used to determine that each of the $90^{\circ}$ plies carry approximately $1.7 \%$ of the total load on the $\left[90 / 0_{3}\right]_{\mathrm{s}}$ laminate. Hence, it can safely be assumed that the contribution of the $90^{\circ}$ plies to the strength and lifetime of the laminate is negligible.

Two batches of the APC-2 laminate are fabricated. Although both batches had the same fiber volume fraction and lay-up, very different strength distributions are measured as shown in Table 1. The reason for this

Table 1

Quasi-static strength of APC- $2 / 90 / 0_{3} / s$ (strengths reported at $76 \mathrm{~mm}$ gage length)

\begin{tabular}{|c|c|c|c|c|}
\hline & \multicolumn{2}{|c|}{ Batch I } & \multicolumn{2}{c|}{ Batch II } \\
\hline & $\begin{array}{l}\tilde{\sigma}_{o}^{\exp } \\
(\mathbf{G P a})\end{array}$ & $\tilde{m}^{\exp }$ & $\begin{array}{c}\tilde{\sigma}_{o}^{\exp } \\
(\mathbf{G P a})\end{array}$ & $\tilde{m}^{\exp }$ \\
\hline $\mathbf{1 2 5}^{\circ} \mathbf{C}$ & 1.56 & 24.7 & 1.78 & 6.6 \\
\hline $140^{\circ} \mathbf{C}$ & 1.42 & 32.3 & 1.69 & 9.0 \\
\hline
\end{tabular}

inconsistency is not clear, although it may be the result of unintended differences in the temperature cycle during processing. The quasi-static strengths of the APC-2 specimens are measured at two temperatures: $125^{\circ} \mathrm{C}$ and $140^{\circ} \mathrm{C}$. All the tests are performed by using a Material Testing System (MTS) servohydraulic machine. The strengths are measured with a loading rate of $445 \mathrm{~N} / \mathrm{sec}$. Since the primary interest is rupture lifetimes, very few specimens are used to measure quasi-static strength. The quasi-static strength of only three specimens is measured at each temperature for the Weibull parameters of Batch I. The quasi-static strength of five specimens is measured at each temperature for the Weibull parameters of Batch II. The rupture lifetimes of the APC-2 specimens are also measured at two temperatures: $125^{\circ} \mathrm{C}$ and $140^{\circ} \mathrm{C}$. The load profile for the tensile rupture tests consists of an initial ramp at $445 \mathrm{~N} / \mathrm{sec}$ and a subsequent hold at the desired load. All the lifetime measurements from both Batch I and Batch II are displayed in Figure 11. However, because of the marked difference in the strength of Batch I and Batch II they are treated separately when calculating $R^{\mathrm{cxp}}$ in Figure 11 . The test is stopped after approximately 4 days, and any specimen that does not fail in that period 


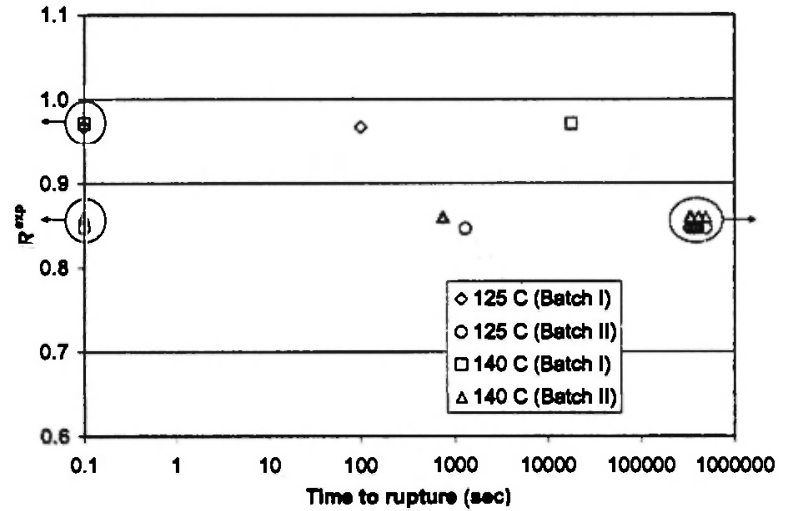

Fig. 11: Stress rupture lifetime of APC-2 $\left[90 / 0_{3}\right]_{\mathrm{s}}$ specimens

of time is treated as a runout. The data points corresponding to instantaneous failures in Figure 11 are placed at 0.1 seconds. It is immediately apparent that there is a very large variability in rupture lifetimes at each stress level. Hence, it is very important that a life prediction technique be stochastic in nature, and be able to compute material reliability at a given stress level and temperature. It will be shown that the Monte Carlo simulation technique described here is particularly well suited to do this.

However, the experimental results for rupture lifetime shown in Figure 11 (and the simulation predictions shown later) should always be interpreted in light of the quasi-static strength distribution for two important reasons. Firstly, the strength distribution can be used to establish the probability of instantaneous failure at a given rupture stress level. Secondly, a material system with greater variability in quasi-static strength may intuitively be expected to possess a greater variability in stress-rupture lifetime. For example, there is a significant variability in the quasi-static strength distribution for Batch II that may translate into greater variability in rupture lifetime measurements for Batch II.

The fiber strength statistics, fiber stiffness and geometry, and the viscoelastic shear properties of the matrix are required to implement the stress rupture simulation described in Section 4.1. Wimolkiatisak and Bell /16/ have studied the strength of Hercules AS4 carbon fibers using the single-fiber fragmentation test. Their data can be used to calculate the following parameters for the Weibull strength distribution of AS4 carbon fibers: $\sigma_{0}=5.25 \mathrm{GPa}, l_{0}=1 \mathrm{~mm}$, and $m=10.65$. The axial Young's modulus of AS4 carbon fibers $E_{f}=$ $234.4 \mathrm{GPa}$, and the fiber radius $r_{f}=3.5 \mu \mathrm{m}$. The shear creep compliance master curve and shift factors shown in Figure 12 are generated from short term creep data of neat PEEK at several temperatures. Flexural mode creep tests were conducted in a TA Instruments Dynamic Mechanical Analyzer (DMA) 2920. It should be pointed out that approximate shear properties are calculated from flexural mode tests in a DMA. Figure 12 gives the shear creep compliance of PEEK over several decades of time at any temperature from $124^{\circ} \mathrm{C}$ to $205^{\circ} \mathrm{C}$. With this information predictions of rupture lifetime can be obtained at any temperature from $124^{\circ} \mathrm{C}$ to $205^{\circ} \mathrm{C}$. Hence, the simulation technique described here can be used to understand and predict the role of temperature in accelerated measurement of stress-rupture lifetimes.

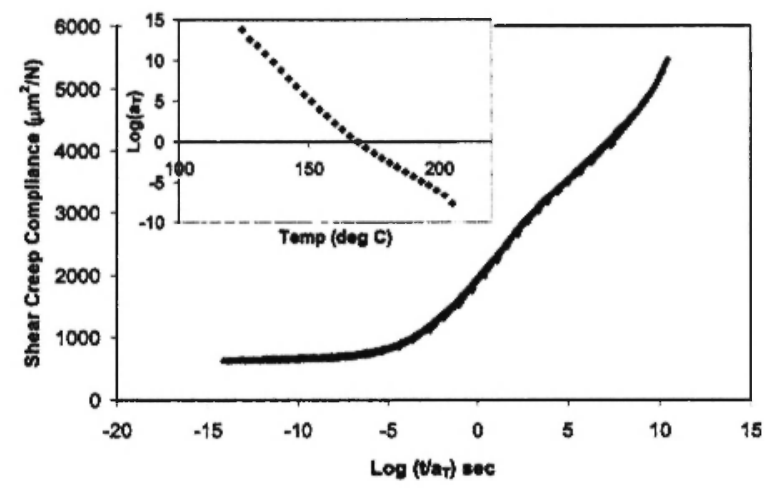

Fig. 12: Master curve and shift factors for shear creep compliance of PEEK

\subsection{Stress-Rupture Simulation Results}

The Monte Carlo simulation approach is used to predict stress rupture lifetimes of the APC- 2 composite at $125^{\circ} \mathrm{C}$ and $140^{\circ} \mathrm{C}$. The material properties described in Section 4.2. are used for the lifetime predictions.

As mentioned earlier, the first step is to compute a quasi-static strength distribution. The strength distribution is required at both $125^{\circ} \mathrm{C}$ and $140^{\circ} \mathrm{C}$. The specialization of load-sharing described in Section 0 for instantaneous ramp up would not predict different quasi-static strengths at different temperatures since it is based on the instantaneous shear compliance of the 
matrix. For temperature dependent strength predictions, it is necessary consider finite ramp up rates with temperature and time dependent viscoelastic shear properties for the matrix material. The general loadsharing approach described in Section 2 cannot be easily modified to account for a finite ramp rate $\alpha$, in the farfield fiber stress, because the Laplace inversion approximation is developed for problems where all inputs are step-functions in time applied at $t=0 / 5 /$. An approximation is used to compute load sharing with a finite ramp rate for far-field fiber stress. The stresses at time $t$ are computed by treating the far-field stress $\alpha t$ as if it were a step-function in time applied at $t=0$ i.e. $\sigma_{f f}^{\mathrm{rtpt}}=\alpha t \mathrm{H}(t)$. With this technique it is possible to use time and temperature dependent matrix shear properties to compute temperature dependent quasistatic strength distributions. However, the loading rate of $445 \mathrm{~N} / \mathrm{sec}$ is high enough that time and temperature depend matrix deformation does not play a significant role in the strength predictions and the same strength distribution is computed at both temperatures. 100 strength computations are performed on a simulation volume consisting of a $10 \times 10$ array of fibers with axial length $X=0.47 \mathrm{~mm}$. The Weibull parameters obtained from the 100 strength values are shown in Table 2. Different strength distributions are obtained by applying NNLS and HVDLS. It should be pointed out that $\tilde{\sigma}_{0}^{\text {sim }}$ is computed for unidirectional APC-2, while the strengths reported in Table 1 are for the $\left[90 / 0_{3}\right]_{s}$ laminate. Since the $90^{\circ}$ plies may be assumed to carry no load, $\bar{\sigma}_{o}^{\operatorname{sim}}$ for the $/ 90 / 0_{3} / \mathrm{s}$ laminate may be assumed to be $3 / 4^{\text {th }}$ of the values reported in Table 2 .

Figure 13 through Figure 16 show rupture lifetime predictions for the APC-2 material. All the rupture lifetime predictions are performed with a $t_{\max }$ of 4 days. The simulation volume consisting of a $10 \times 10$ array of

Table 2

Quasi-static strength predictions of unidirectional APC$2 V_{f}=54 \%$ obtained by applying two different loadsharing techniques (strengths reported at $X=0.47 \mathrm{~mm}$ )

\begin{tabular}{|c|c|c|}
\hline & $\tilde{\sigma}_{o}^{\text {sim }}$ & $\tilde{m}^{\text {sim }}$ \\
\hline NNLS & 2.60 & 47.5 \\
\hline HVDLS & 2.72 & 80.1 \\
\hline
\end{tabular}

fibers with axial length $X=0.47 \mathrm{~mm}$.

Figure 13 and Figure 14 are the rupture lifetimes predictions at $125^{\circ} \mathrm{C}$ and $140^{\circ} \mathrm{C}$, respectively, calculated with NNLS. The rupture lifetime predictions are plotted along with the experimental results. At each $R^{\text {cxp }}, 100$ rupture lifetimes are comsputed. The numbers displayed with the symbol ' $\longleftarrow$ ' represent experimental and predicted instantaneous failures. The symbol ' $\rightarrow$ ' and its associated number represent experimental and predicted runouts in 4 days. The number of measured and predicted runouts may be regarded as the experimental and predicted reliability of the material to withstand the given stress level and temperature for a desired lifetime of 4 days. The horizontal brace and its associated number refer to the measured and predicted rupture failures that occur within 4 days. $R^{\text {sim }}$, and hence, the far-field fiber stress level for the rupture simulation is calculated by equating the experimental probability of instantaneous failure with the probability of instantaneous failure for the simulation volume as given by Equation (2). The number of instantaneous

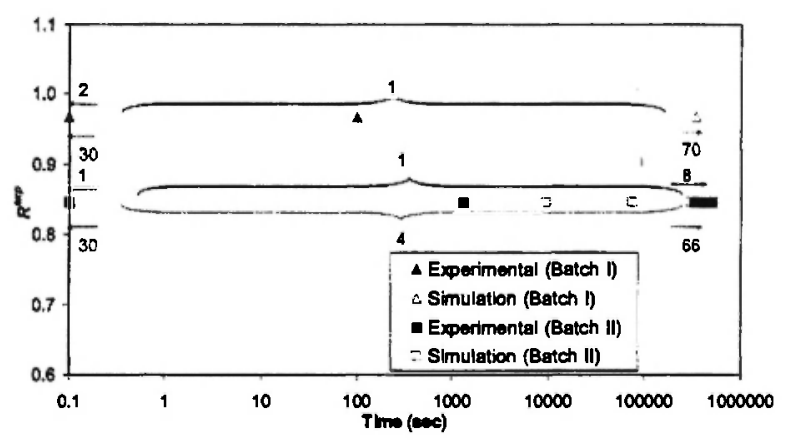

Fig. 13: Rupture lifetime predictions for APC-2 composite at $125^{\circ} \mathrm{C}$ (NNLS)

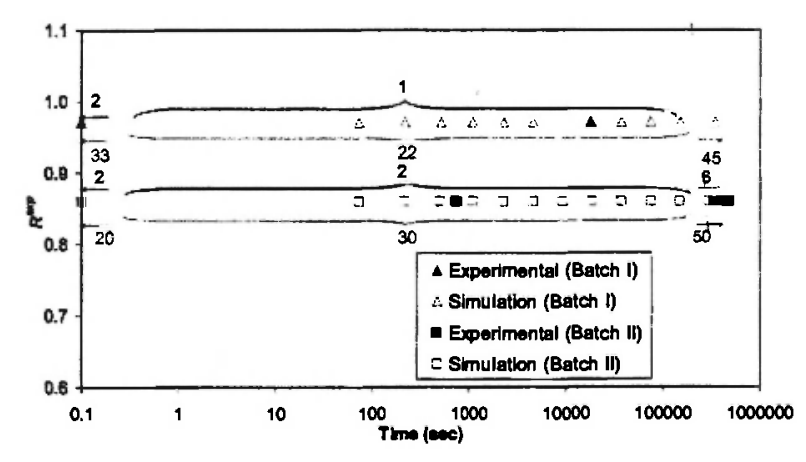

Fig. 14: Rupture lifetime predictions for APC-2 composite at $140^{\circ} \mathrm{C}$ (NNLS) 
failures predicted by the simulation as a fraction of 100 is approximately equal to the probability of instantaneous failure at each stress level and temperature. Since more experimental measurements are made for Batch II, a complete comparison is obtained at the lower $R^{\text {exp }}$ in Figure 13 and Figure 14. The simulations predict more runouts at the lower temperature of $125^{\circ} \mathrm{C}$ than at $140^{\circ} \mathrm{C}$. Consequently, far fewer rupture failures are predicted at $125^{\circ} \mathrm{C}$ than at $140^{\circ} \mathrm{C}$. Although, the experimental measurements are limited, they appear to have a similar trend of longer lifetimes at $125^{\circ} \mathrm{C}$ than at $140^{\circ} \mathrm{C}$. It is encouraging to note that at fraction of instantaneous failures, rupture failures in 4 days, and runouts show a close correlation between the measurements and predictions at $140^{\circ} \mathrm{C}$ and the lower $R^{\exp }$.

1. Similarly, the rupture lifetimes computed with HVDLS at $125^{\circ} \mathrm{C}$ and $140^{\circ} \mathrm{C}$ are shown in Figure 15 and Figure 16, respectively. The HVDLS also predicts longer lifetimes at lower temperatures. Comparing the

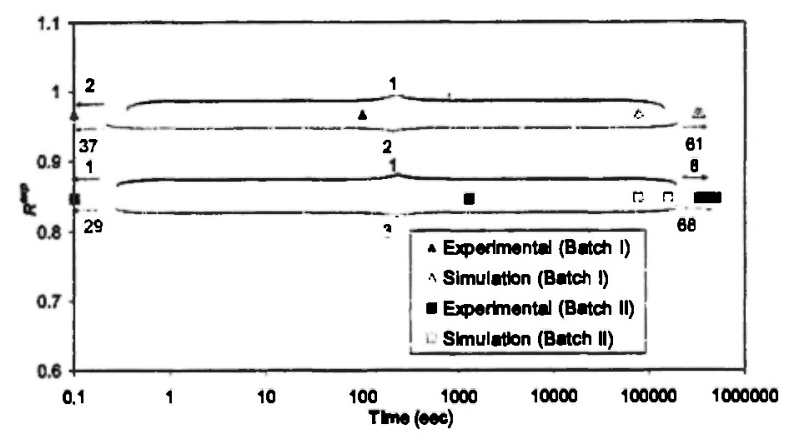

Fig. 15: Rupture lifetime predictions for APC-2 composite at $125^{\circ} \mathrm{C}$ (HVDLS)

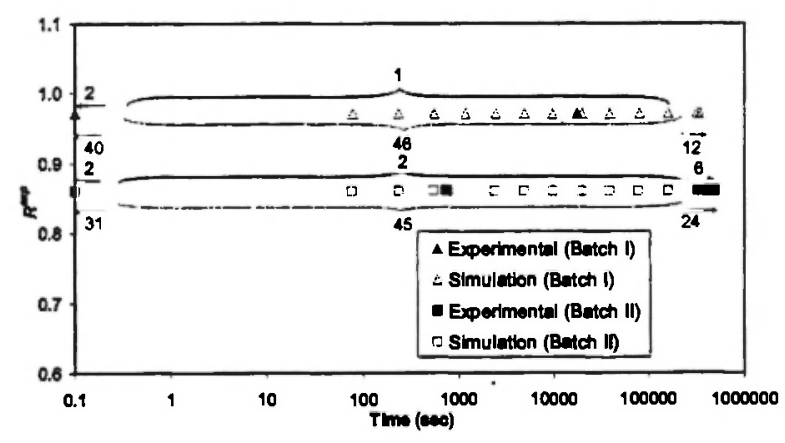

Fig. 16: Rupture lifetime predictions for APC-2 composite at $140^{\circ} \mathrm{C}$ (HVDLS) rupture lifetimes obtained by NNLS and HVDLS, it appears as if the HVDLS technique predicts shorter lifetimes. As explained in Section 3, for HVDLS a single fractured fiber perturbs the stresses in all the neighboring fibers and there is an increase in the overstressed region of all the unbroken fibers with time. For NNLS, only the stresses in the nearest unbroken neighbors is perturbed due to single fractured fiber. Hence, stress rupture lifetimes computed with NNLS are longer than lifetimes computed with HVDLS.

\section{SUMMARY AND CONCLUSIONS}

This paper describes a micromechanical technique for predicting the lifetime of unidirectional polymer composites loaded under tensile stress-rupture conditions. It is assumed that stress rupture in unidirectional composite materials occurs as a result of viscoelastic deformation in the matrix. The timedependent response of the matrix causes an increase in the overstressed length on unbroken fibers near a cluster of fiber fractures. This increases the probability of failure of the unbroken fibers, and consequently the probability of failure of the composite material as a whole. The formulation presented in this work assumes linearly viscoelastic matrix behavior.

The first step in this effort is to develop a general framework for micromechanical stress redistribution due to an arbitrary sequence of fiber fractures. An approximate technique using Boltzmann superposition of time-dependent influence functions is utilized. Two different sets of influence-functions are calculated based on different shear-lag load-sharing assumptions. The first set of influence functions assume that the load of a broken fiber is redistributed only onto the nearest neighbors. This form of load sharing is termed Nearest Neighbor Load Sharing (NNLS) and it is developed because model composite experiments and finite element analysis of micromechanical load redistribution show that NNLS is applicable under quasi-static conditions $16 \%$ The second set of time-dependent influence functions are developed by extending the traditional quasi-static analysis of Hedgepeth and Van Dyke $/ 2 /$. A favorable comparison is made between the time-dependent load sharing analysis and measurements 
of the strain redistribution in model composites.

The load sharing framework is incorporated into a Monte Carlo simulation to predict the stress rupture lifetime of unidirectional composite materials. An encouraging comparison is made between predicted and measured lifetimes of a $\left[90 / 0_{3}\right]_{\mathrm{s}}$ APC-2 composite laminate at $125^{\circ} \mathrm{C}$ and $140^{\circ} \mathrm{C}$. Long-term time and temperature dependent viscoelastic properties of the matrix material are easily obtained by applying timetemperature superposition principles to short-term creep or relaxation data measured in a dynamic mechanical analyzer at several temperatures. This information is supplied to the simulation to predict long-term rupture lifetimes at any temperature. In this manner the simulations help understand and predict the role of temperature in accelerated measurement of stress rupture lifetimes. The extreme variability in rupture lifetimes makes it very important for predictive techniques to be able to assess composite reliability for a desired lifetime at a given stress level and temperature. The Monte Carlo simulation approach is particularly well suited to determine reliability under stress rupture conditions.

Measuring the stress-rupture lifetime of purely unidirectional composites is challenging because initial damage occurs in the gripped section of the specimen. The rupture lifetime of a unidirectional specimen is very sensitive to the number and location of the initial fiber fractures. Large initial damage in the gripped section necessarily leads to ultimate material failure in the gripped section. This paper puts forward recommendations to alleviate this problem by testing specimens with a $\left[90 / 0_{\mathrm{n}}\right]_{\mathrm{s}}$ layup. The surface $90^{\circ}$ plies protect the $0^{\circ}$ layers from damage in the grip section while at the same time carrying a negligible fraction of the total load. Consistent gage section failures are observed when testing the $\left[90 / 0_{3}\right]_{\mathrm{s}}$ APC-2 composite specimens.

\section{ACKNOWLEDGEMENTS}

The authors appreciate Professor K. L. Reifsnider's recommendations for improvement of this work. This work was supported by the National Science Foundation and the Air Force Office of Scientific Research (NSF Grant No. CMS-9872331), and the Army Research Office (ARO Grant No. 42451-EG).

\section{REFERENCES}

1. J.M. Hedgepeth, Stress concentrations in filamentary structures. NASA TN D-882, Langley Research Center, 1961.

2. J.M. Hedgepeth and P. Van Dyke, Local stress concentrations in imperfect filamentary composite materials. Journal of Composite Materials 1, 294309 (1967).

3. D.C. Lagoudas, C. Hui and S.L. Phoenix, Time evolution of overstress profiles near broken fibers in a composite with a viscoelastic matrix. International Journal of Solids and Structures 25(1), 45-66 (1989).

4. I.J. Beyerlein, S.L. Phoenix and R. Raj, Time evolution of stress redistribution around multiple fiber breaks in a composite with viscous and viscoelastic matrices. International Journal of Solids and Structures 35 (24), 3177-3211 (1998).

5. R.A. Schapery, Stress analysis of viscoelastic composite materials. Journal of Composite Materials 1, 228-267 (1967).

6. T. Bandorawalla, Micromechanics-Based Strength and Lifetime Prediction of Polymer Composites, Ph.D. Dissertation, Virginia Polytechnic Institute and State University, Available electronically at http://scholar.lib.vt.edu/theses/available/etd03172002-151608/ (2002).

7. J.M. Lifshitz and A. Rotem, Time-dependent longitudinal strength of unidirectional fibrous composites. Fibre Science and Technology, 3, 1-20 (1970)

8. J.M. Lifshit,. MED Report No. 33, Department of Materials Engineering, Technion, Israel.

9. M. Ibnabdeljalil and S.L. Phoenix, Creep rupture of brittle matrix composites reinforced with time dependent fibers: scalings and Monte Carlo simulations. Journal of the Mechanics and Physics of Solids 43 (6), 897-931 (1995). 
10. N. Iyengar and W.A. Curtin, Time-dependent failure in fiber-reinforced composites by fiber degradation. Acta Materialia 45 (4), 1489-1502 (1997a).

11. N. Iyengar and W.A. Curtin, Time-dependent failure in fiber-reinforced composites by matrix and interface shear creep. Acta Materialia 45 (8), 3419-3429 (1997b).

12. Z.Z. Du and R.M. McMeeking, Creep models for metal matrix composites with long brittle fibers. Journal of the Mechanics and Physics of Solids 43(5), 701-726 (1995).

13. S.L. Phoenix, P. Schwartz and H.H. Robinson IV, Statistics for the strength and lifetime in creep rupture of model carbon/epoxy composites.
Composites Science and Technology 32, 81-120 (1988).

14. H. Otani, S.L. Phoenix and P. Petrina, Matrix effects on lifetime statistics for carbon fiber-epoxy microcomposites in creep rupture. Journal of Materials Science 26, 1955-1970 (1991).

15. C.M. Landis, I.J. Beyerlein and R.M. McMeeking, Micromechanical simulation of the failure of fiber reinforced composites. Journal of the Mechanics and Physics of Solids 48, 621-648 (2000).

16. A.S. Wimolkiatisak and J.P. Bell, Interfacial shear strength and failure modes of interphase-modified graphite-epoxy composites. Polymer Composites 10 (3), 162-172 (1989). 
